\title{
Comparison of brief clinical delirium and cognitive testing among patients admitted via the trauma and orthopaedic acute intake - a service evaluation on the clinical dependence, efficacy and accessibility of implementing Gwent orientation and awareness listing testing in relation to the 4AT at the Royal Gwent Hospital, Newport
}

\author{
Authors: Jack Wellington, ${ }^{A}$ Alexander Eggleton, ${ }^{B}$ Reem Naji, ${ }^{B}$ Miles Allison ${ }^{C}$ and Inderpal Singh ${ }^{C}$
}

\begin{abstract}
Introduction
Gwent orientation and awareness listing (GOAL) is the brief delirium/cognitive clinical test currently employed at Royal Gwent Hospital, Newport, whereas the 4AT test is routinely utilised at Ysbyty Ystrad Fawr Hospital. ${ }^{1,2}$ To our knowledge, both tests have not been prospectively compared relative to equal patient cohorts. We aim to evaluate two rapidly performed valid cognitive examinations among the same patient cohort, and assess patient testing results among acute/emergency trauma and orthopaedic (T\&O) admissions compared with previously obtained patient data presenting via the acute medical intake.
\end{abstract}

\section{Materials and methods}

Verbal consent to cognitive testing by means of GOAL and $4 A T$ was sought from patients presenting acutely to $T \& O$ admissions over a 4 -week period. A GOAL score of $<8 / 10$ is deemed a 'fail', and on 4AT any error is deemed 'possible cognitive impairment'.,2 Patient documentation regarding dementia, epilepsy and psychiatric/ neurological illness was recorded alongside living arrangements.

\section{Results and discussion}

There were 146 patients, of whom 10 were not well enough to be scored, and one patient declined to participate. Of the 135 participants, 92 passed both GOAL and 4AT (68.15\% overall success rate; GOAL average score $=9.31$; 4 AT average score $=0$ ), 40 failed the 4 AT $(29.63 \%$ 4AT failure rate; average score $=$ 2.45), 21 failed the GOAL (15.56\% GOAL failure rate; average

Authors: ${ }^{A}$ Cardiff University School of Medicine, Cardiff, Wales, UK; ${ }^{B}$ Cardiff University, Cardiff, Wales, UK; ${ }^{C}$ Aneurin Bevan University Health Board, Wales, UK score $=5.43)$ and 18 failed both the GOAL and 4 AT $(13.33 \%$ overall failure rate; $\mathrm{GOAL}$ average score $=5.22$; $4 \mathrm{AT}$ average score $=3.17$ ). Also observed were three participants who failed the GOAL but passed on the 4AT. Hence the probability of a participant failing the 4AT was significantly greater than with the GOAL (chi-squared $=7.65 ; p<0.01$ ). Likelihood of test failure was significantly greater with 4 AT (chi-squared $=7.65 ; p<0.01$ ). Ages and comparisons on GOAL testing results with a historical general medical patient cohort displayed significant differences between patient cooperation in acute medical and $T \& O$ intakes. The median age of eligible study participants are 64 (standard deviation (SD) 19.8) and 73 (SD 18) years of age, with 114 $(84 \%)$ and $720(73 \%)$ participants obtaining the pass score of $>7$ points, and $21(16 \%)$ and $270(27 \%)$ participants who failed testing (threshold of $<8$ points) within $T \& O$ and medical intakes respectively. We also observed the number of participants unable to cooperate ( 0 and 58 patients for T\&O and medical intakes respectively). In addition, of the 135 eligible participants in the $T \& O$ cohort, we documented a number of following conditions/comorbidities present in individuals included in the current study; five with known or diagnosed dementia, six with a background of alcohol dependence, 28 with psychiatric diagnoses, three with known or diagnosed epilepsy and one with a known learning disability.

\section{Conclusion}

The 4AT test is more likely to signal cognitive impairment than GOAL among T\&O emergency admissions; and T\&O intake patients are more likely to cooperate with cognitive testing by GOAL, and they perform better than acute medical emergency admissions. Further studies would include analysing the current study's participant deprivation indexes via their documented postcodes to identify whether socioeconomic factors influence GOAL and $4 A T$ failure rates and incidences in cognitive impairment and/or 
delirium, further comparing the GOAL system to other commonly used cognitive testing in other neighbouring health boards and introducing GOAL into primary healthcare environments to assess its efficacy and accessibility within the community.

\section{Conflicts of interest}

None declared.

\section{References}

1 Allison MC, Kontoyannis A, Durai D, Turner GI, Fone DL. GOAL: a simplified mental test for emergency medical admissions. QJM 2004;97:663-9.

2 Shenkin SD, Fox C, Godfrey M et al. Protocol for validation of the 4AT, a rapid screening tool for delirium: a multicentre prospective diagnostic test accuracy study. BMJ Open 2018;8:e015572. 